\title{
Should endobronchial ultrasonography be part of the thoracic surgeon's armamentarium?
}

\author{
Sebastien Gilbert, MD, ${ }^{\mathrm{a}}$ David O. Wilson, MD, MPH, ${ }^{\mathrm{b}}$ Neil A. Christie, MD, ${ }^{\mathrm{a}}$ James D. Luketich, MD, ${ }^{\mathrm{a}}$ Rodney J. Landreneau, MD, ${ }^{\mathrm{a}}$ \\ John M. Close, MS, PMSD, ${ }^{c}$ and Matthew J. Schuchert, MD ${ }^{\mathrm{a}}$
}

\begin{abstract}
Objective: The study objective was to determine the clinical usefulness and accuracy of endobronchial ultrasound-guided needle aspiration of mediastinal and hilar lymph nodes.
\end{abstract}

\begin{abstract}
Methods: A retrospective analysis of a thoracic surgery unit's experience was performed.
Results: In a period of 19 months, 75 patients underwent the procedure (mean age $=65.5 \pm 1.6$ years; male to female $=2: 1$ ) most commonly for mediastinal lymphadenopathy in the setting of diagnosed or suspected lung cancer. It was diagnostic in $68.9 \%$ after rapid on-site evaluation and $74.3 \%$ after final cytologic examination. The rapid on-site evaluation and final cytology results were discordant in $16.2 \%(P<.001)$. In 50 cases, the needle aspirate cytology could be compared with pathology results. The sensitivity and specificity for the diagnosis of cancer were $85 \%$ and $100 \%$, respectively. The false-negative rate endobronchial ultrasound cytology was $8.1 \%$. Mediastinal lymph node station 7 was most commonly biopsied. The stations with the highest diagnostic yield were: $11 \mathrm{R}, 3,10 \mathrm{~L}$, and 7 . Of the patients with a positive positron emission tomography scan with suspected clinical stage III lung cancer, cancer was downstaged in $40 \%$ after endobronchial ultrasound.
\end{abstract}

Conclusion: Endobronchial ultrasound-guided needle aspiration is a clinically useful minimally invasive option for lung cancer staging and evaluation of mediastinal lymphadenopathy. The procedure should be considered complementary to mediastinoscopy.

To date, thoracic surgeons have played an important role in the evaluation of mediastinal lymphadenopathy and the mediastinal staging of lung cancer. This is in large part due to their ability to safely and reliably perform mediastinoscopy. Endobronchial ultrasound-guided transbronchial needle aspiration (EBUS-TBNA) is a relatively new procedure that is often associated with the emerging field of interventional bronchoscopy. Such technology may result in significant changes to the established approaches to diagnosis and management of thoracic diseases. As more efficacy data become available, EBUS may assume an increasingly important role in the practice of thoracic surgery. Through learning and adaptation, thoracic surgeons will likely continue to expand their therapeutic armamentarium while playing a key role in critically appraising new technology. Our main objectives were to successfully integrate EBUS into our minimally invasive surgical practice and obtain data on its clinical usefulness and accuracy for the evaluation of mediastinal lymph nodes.

\footnotetext{
From the University of Pittsburgh, Heart, Lung and Esophageal Institute, ${ }^{\text {a }}$ Division of Pulmonary, Allergy, and Critical Care Medicine, ${ }^{b}$ and School of Dental Medicine, ${ }^{c}$ University of Pittsburgh, Pittsburgh, Pennsylvania.

Received for publication May 2, 2008; revisions received Aug 19, 2008; accepted for publication Sept 16, 2008.

Address for reprints: Sebastien Gilbert, MD, UPMC Presbyterian, Suite C-800, 200

Lothrop St, Pittsburgh, PA 15213 (E-mail: gilberts@upmc.edu).

J Thorac Cardiovasc Surg 2009;137:413-8

$0022-5223 / \$ 36.00$

Copyright (C) 2009 by The American Association for Thoracic Surgery

doi:10.1016/j.jtcvs.2008.09.042
}

\section{MATERIALS AND METHODS}

\section{Patients and Personnel}

With approval from the institutional review board, a retrospective review of all patients who underwent EBUS-TBNA from October 2006 to April 2008 was completed. Completion of a continuing medical education-approved EBUS course and 5 proctored cases were required to be granted privileges to perform the procedure independently. On-site training was provided to the technicians, therapists, nurses, and doctors involved.

\section{Endobronchial Ultrasound Procedure}

The technique and equipment necessary to perform EBUS-TBNA have already been described extensively. ${ }^{1}$ Briefly, procedures were performed either transorally with topical anesthesia and monitored intravenous sedation or under general anesthesia with endotracheal intubation. The instrumentation used was manufactured by Olympus (Center Valley, Pa) and included the Evis Exera ultrasonic bronchoscope model BF-UC160F-OL8, the Evis Exera II video processor model CV-180, the Evis Exera II xenon light source model CLV-80, the EUS Exera CLA processor model EU-C60, and 22-gauge aspiration needles with syringe model NA-201SX-4022-A. During ultrasound examination of a lymph node station, the largest identified lymph node amenable to needle puncture was biopsied. A different aspiration needle was used at each lymph node station to avoid potential specimen contamination. All procedures were performed with a cytologist immediately available for rapid on-site evaluation (ROSE) of transbronchial needle aspirates. ROSE was performed on the first specimen retrieved from the aspiration needle after it had been air-dried and stained with Dif-Quick (American Scientific Products, McGaw Park, Ill). Another slide was prepared from the same initial specimen but was fixed in alcohol and processed at a later time using Papanicolaou staining. The remainder of the specimen was retrieved from the needle by reinserting the guide wire and flushing with preservation solution. It was later centrifuged, and the solid component was embedded in paraffin and processed using histology and immunohistochemistry techniques (eg, cytokeratin-7, cytokeratin-20, and thyroid transcription factor-1). 


\section{Abbreviations and Acronyms \\ $\mathrm{CT}=$ computed tomography \\ EBUS $=$ endobronchial ultrasound-guided transbronchial needle aspiration \\ PET = positron emission tomography \\ ROSE $=$ rapid on-site evaluation}

or hilar lymph node(s) on positron emission tomography (PET) scan without lymphadenopathy $(\mathrm{n}=16 ; 21.4 \%)$, mediastinal staging (ie, no lymphadenopathy and negative PET scan) $(n=6 ; 8 \%)$, and other $(n=1 ; 1.3 \%)$. There were no complications, and all patients were discharged within 24 hours of the procedure ( $98.7 \%$ on the same day).

PET/computed tomography (CT) scans were obtained before EBUS-TBNA in $70.6 \%(\mathrm{n}=53)$, and $81.1 \%$ of the scans $(n=43)$ were positive in mediastinal or hilar lymph nodes. In patients with a negative PET scan $(\mathrm{n}=10)$, EBUS-TBNA was negative in 7 and nondiagnostic in 3. After subsequent pathologic testing $(\mathrm{n}=8)$, EBUS-TBNA was positive for cancer in 1 of the patients with a negative PET scan. Of the patients with a positive PET scan $(n=43)$, EBUS-TBNA was positive for cancer in $13(30.2 \%)$, negative in $16(37.2 \%)$, and nondiagnostic in $14(32.6 \%)$. Pathologic testing was available in 29 patients with positive PET scans; of these, $15(34.9 \%$; 15/43) were positive for cancer and $14(32.6 \% ; 14 / 43)$ were negative. On the basis of a positive mediastinal PET scan, clinical stage III lung cancer was suspected in 20 patients with no history of malignancy. In these patients, EBUS-TBNA was negative in $10(50 \%)$, positive in $6(30 \%)$, and nondiagnostic in $4(20 \%)$. Of the 10 patients in whom EBUS was negative, 2 had positive mediastinal lymph nodes after mediastinoscopy.

\section{Lymph Node Stations}

The number of lymph node stations sampled was 1 in $56 \%(\mathrm{n}=42), 2$ in $24 \%(\mathrm{n}=18), 3$ in $18.7 \%(\mathrm{n}=14)$, and 4 in $1.3 \%(\mathrm{n}=1)$. The lymph node stations sampled were station $7(\mathrm{n}=42 ; 56 \%)$, station $4 \mathrm{R}(\mathrm{n}=29$; $38.7 \%)$, station $10 \mathrm{R}(\mathrm{n}=20 ; 26.7 \%)$, station $4 \mathrm{~L}(\mathrm{n}=15$; $20 \%)$, station 10L $(\mathrm{n}=7 ; 9.3 \%)$, station $3(\mathrm{n}=7$; $9.3 \%)$, station $11 \mathrm{R}(\mathrm{n}=4 ; 5.3 \%)$, and station $11 \mathrm{~L}(\mathrm{n}=$ $1 ; 1.3 \%$ ). Stations $2 \mathrm{R}$ and $2 \mathrm{~L}$ were not sampled in any of the patients. Table 1 summarizes the diagnostic yield at mediastinal stations that were sampled in 10 patients or more. The concordance column reflects the proportion of patients in whom the results were the same on ROSE and final EBUS cytology. The overall mean number of needle passes used to sample lymph nodes was $2.1 \pm 0.1$ (range, 1-6). The diagnostic yield ranged from $58.6 \%$ to $100 \%$, and the stations with the highest yields were $11 \mathrm{R}, 3,10 \mathrm{~L}$, and 7 . For all the sampled stations, there was complete agreement between ROSE and final cytology when the lymph nodes were positive for cancer. When the ROSE was negative, cytology results were concordant in $85.7 \%$ to $100 \%$. In the most commonly sampled lymph node stations (Table 1), final cytologic examination yielded a diagnosis in $12.5 \%$ to $50 \%$ of patients in whom ROSE was deemed nondiagnostic. At the time of final cytologic examination, the cytopathologist has access to additional biopsy material in the form of a slide smear stained with the Papanicolaou method and cell block preparations from centrifuged biopsy material. lymphadenopathy $(\mathrm{n}=52 ; 69.3 \%)$, positive mediastinal 
TABLE 1. Diagnostic yield and concordance between ROSE and final cytology at specific lymph node stations

\begin{tabular}{|c|c|c|c|c|c|c|c|}
\hline \multirow[b]{2}{*}{ Station } & \multirow[b]{2}{*}{$\mathbf{n}^{\mathbf{a}}$} & \multirow[b]{2}{*}{ Needle passes (mean) } & \multicolumn{2}{|c|}{ EBUS cytology } & \multicolumn{3}{|c|}{ Concordance $^{b}$} \\
\hline & & & Diagnostic & ND & ROSE Positive & ROSE Negative & ROSE ND \\
\hline $4 \mathrm{R}$ & 29 & $2.3 \pm 0.2$ & $62.1 \%(18)$ & $38.9 \%(11)$ & $100 \%$ & $92.9 \%$ & $75 \%$ \\
\hline $4 \mathrm{~L}$ & 15 & $2.1 \pm 0.3$ & $73.3 \%(11)$ & $26.7 \%$ & $100 \%$ & $100 \%$ & $50 \%$ \\
\hline 7 & 41 & $2.2 \pm 0.2$ & $78 \%(32)$ & $22 \%(9)$ & $100 \%$ & $85.7 \%$ & $53.3 \%$ \\
\hline $10 \mathrm{R}$ & 20 & $2.0 \pm 0.3$ & $60 \%(12)$ & $40 \%(8)$ & $100 \%$ & $100 \%$ & $87.5 \%$ \\
\hline
\end{tabular}

EBUS, Endobronchial ultrasound; ROSE, rapid on-site evaluation; $N D$, nondiagnostic. ${ }^{\mathrm{a}}$ Number of patients in whom lymph node station was sampled. ${ }^{\mathrm{b}}$ Represents the proportion of patients in whom ROSE results were the same as final EBUS cytology results.

This may explain the observed difference in diagnostic yield between ROSE and final cytology.

\section{Diagnostic Performance}

ROSE results were recorded in 74 patients and compared with final cytology results. The ROSE and final cytology results were different in $16.2 \%$ of patients $(P<.001)$. It should be noted that $30.4 \%$ of nondiagnostic ROSE results were eventually found to be negative $(17.4 \%)$ or positive for cancer $(13 \%)$ after final EBUS cytologic evaluation. In the group with negative ROSE results, $92.9 \%$ ended up negative after final cytology. All patients with positive results for cancer after ROSE also had positive results for cancer on final cytology (ie, $100 \%$ correlation). In the 3 patients $(4.1 \%)$ in whom ROSE showed atypical cells, the cytology results were evenly distributed between nondiagnostic, negative, and positive for cancer. Overall, EBUS-TBNA was diagnostic in $68.9 \%$ after ROSE and $74.3 \%$ after final cytologic evaluation.

In two thirds of patients $(\mathrm{n}=50)$, the results of EBUSTBNA could be compared with mediastinoscopy $(n=34$; $45.3 \%)$, immunohistochemistry $(\mathrm{n}=11 ; 14.7 \%)$, or intraoperative mediastinal lymph node sampling $(n=5 ; 6.7 \%)$. In the remaining 25 patients, additional biopsies were not obtained after EBUS for the following reasons: single or multiple lymph node stations found positive for cancer $(\mathrm{n}=5$; $20 \%$ ), specific benign diagnosis obtained (eg, sarcoidosis) $(\mathrm{n}=5 ; 20 \%)$, not a surgical candidate or declined additional procedures $(n=4 ; 16 \%)$, previous mediastinoscopy $(n=4$; $16 \%$ ), abnormal lymph node not accessible by mediastinoscopy $(\mathrm{n}=2 ; 8 \%)$, and other $(\mathrm{n}=5 ; 20 \%)$. When the EBUS cytology was nondiagnostic $(n=13 ; 24 \%)$, the pathology was benign in $61.5 \%(\mathrm{n}=8)$ and malignant in $38.5 \%(\mathrm{n}=5)$. Three of 18 patients $(16.6 \%)$ with benign cytology were eventually found to have cancer. In all patients in whom EBUS-TBNA cytology was positive for cancer, the pathologic testing was also positive for cancer. More specifically the cytologic diagnosis was correct in $90 \%$ of non-small cell lung cancers, $100 \%$ of small cell lung cancers and metastatic cancers from a primary other than the lung, and $50 \%$ of sarcoidosis cases. In the subgroup of patients with a diagnostic EBUS-TBNA $(\mathrm{n}=37)$, the sensitivity, specificity, positive predictive value, and negative predictive value for the detection of cancer were $85 \%$, $100 \%, 100 \%$, and $85 \%$, respectively. The false-negative rate of EBUS-TBNA cytology was $8.1 \%$.

\section{Diagnostic Failures}

The patient's diagnosis or the indication to perform EBUS-TBNA did not have a significant influence on the overall diagnostic success rate. The presence or absence of lymphadenopathy on CT scan and the PET findings (positive or negative), analyzed alone or in all possible combinations, did not influence the probability of obtaining diagnostic cytology. There was no significant difference in the average number of lymph node stations biopsied between the diagnostic and nondiagnostic EBUS cases. Overall, there was no threshold number of procedures after which the diagnostic rate improved. This was also true for 2 of the operators who each performed more than 20 procedures. The probability of failure to obtain a tissue diagnosis was also independent of the operator's qualifications (surgeon vs pulmonologist).

\section{DISCUSSION}

In small peripheral lung tumors with no lymphadenopathy, a negative mediastinal PET or PET/CT scan may be helpful in guiding a selective approach to mediastinoscopy. The probability that such patients have mediastinal lymph node metastases is low. ${ }^{2}$ On the other hand, PET scanning has a significant rate of false-positive results for mediastinal lymph node metastases in patients with lung cancer. ${ }^{3}$ Therefore, it may be best to confirm positive mediastinal PET findings with a lymph node biopsy. This course of action should minimize stage misclassification and ensure that surgical candidates are not denied potentially curative resection. In patients with a positive mediastinal PET scan, EBUS may be helpful in determining whether lymph nodes are truly involved with cancer. If the EBUS-TBNA is positive for cancer, mediastinoscopy may not be necessary as illustrated in Figure 1. Our preliminary experience seems to support the use of EBUS-TBNA as complementary to mediastinoscopy in obtaining diagnostic tissue from mediastinal lymph nodes. Some investigators may be of the opinion that EBUS, in combination with endoscopic esophageal ultrasound, may eventually render mediastinoscopy obsolete. ${ }^{4}$ They 


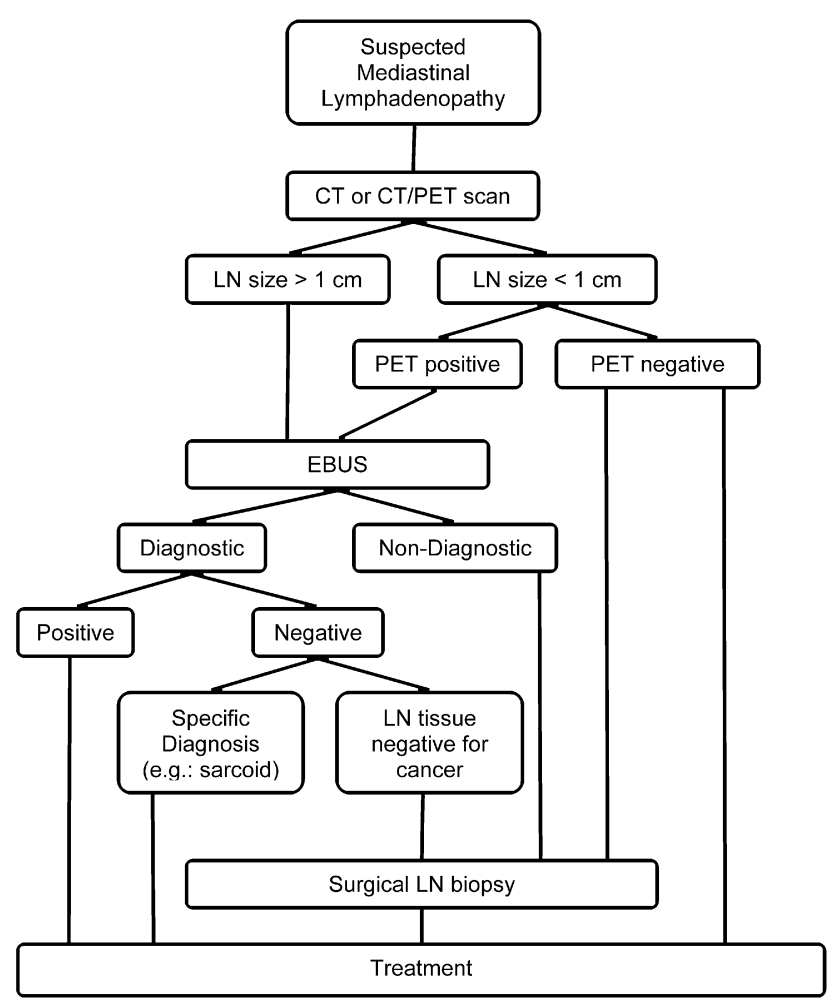

FIGURE 1. Diagnostic approach to the patient with suspected malignant mediastinal lymphadenopathy. $C T$, Computed tomography; $P E T$, positron emission tomography; $L N$, lymph node; EBUS, endobronchial ultrasound.

may quote that as many as $54.4 \%$ of mediastinoscopies yield no lymphoid tissue on final pathology. ${ }^{5}$ However, this seemingly high proportion would be more accurately interpreted in light of the qualifications of the surgeons performing mediastinoscopy. Unfortunately, this information is not available. The yield of mediastinoscopy seems to be substantially higher in large case series (ie, $>3000$ patients) published by thoracic surgeons. ${ }^{6,7}$

The probability of a false-negative frozen section or final pathology result after mediastinoscopy is reportedly low $(0.6 \%-8.8 \%))^{6,8}$ Therefore, it is considered acceptable to perform mediastinoscopy either as a stand-alone procedure or in combination with pulmonary resection. To evaluate EBUS-TBNA as an intraoperative decision-making tool, we reviewed our ROSE results. The purpose of ROSE is to minimize inadequate specimens and improve diagnostic yield. In this study, the proportion of nondiagnostic ROSE results was higher than previously published rates after TBNA $(31.1 \%$ vs $3.3 \%-17.8 \%){ }^{9,10}$ However, this may be related in part to the relatively lower prevalence of cancer in our study population ( $44 \%$ vs $66-71.6 \%$ ). On the basis of our results and other reports, we have decided not to use intraoperative (ie, ROSE) EBUS-TBNA results to decide whether it is indicated to proceed with lung resection.

The final cytology report is usually available within a few days of the procedure. In previously published larger (ie, $n>100)$ EBUS case series, the sensitivity, specificity, positive predictive values, and negative predictive values were $92.3 \%$ to $94.6 \%, 100 \%, 100 \%$, and $11 \%$ to $97.4 \%$, respectively. ${ }^{11-13}$ Although only a subset of our patients $(66.6 \%)$ had pathologic testing in addition to EBUS cytology, we found this proportion to be comparable to published literature $(6 \%-100 \%) .{ }^{11-13}$ Although in our study population, the specificity $(100 \%)$ was similar to published data, the sensitivity $(85 \%)$ was slightly lower. The diagnostic yield of cytology was also lower in our study $(74.3 \%$ vs $93.5 \%-100 \%)^{11-13}$ Once again, the lower sensitivity and diagnostic accuracy in our study group may be due to differences in disease prevalence between study populations ( $44 \%$ vs $25.5 \%-100 \%$ ). Other potential factors (ie, operator, number of cases, diagnosis, indication, preoperative imaging, and number of stations sampled) did not have a significant influence on diagnostic accuracy.

We realize that the results must be interpreted in light of the limitations inherent to a retrospective study design. We also acknowledge that generalizations regarding diagnostic performance may be limited by the need for subset analysis. Because this was not a prospective research protocol, mediastinoscopy was performed when judged clinically appropriate. At this point in our experience, the results lend support to the use of mediastinoscopy when EBUS-TBNA is negative or nondiagnostic. In selected patients where the cytology is positive for cancer, a specific benign diagnosis is obtained, the operator is confident that the scope was positioned accurately, and the needle puncture sites are in the correct anatomic location, it may be reasonable to forego mediastinoscopy. The relationship between EBUS and mediastinoscopy will likely be refined once more data are available.

\section{CONCLUSIONS}

Our results have guided the design of a clinical pathway for the use of EBUS in the evaluation of patients with abnormal mediastinal lymph nodes on CT or PET scan (Figure 1). Acquired experience with EBUS provides preliminary data and mitigates the potential negative effects of procedural training and learning curve on diagnostic performance. Although the actual needle sampling technique is relatively easy to master, it is our impression that a significant period of adaptation and learning is necessary to understand endobronchial ultrasonography of the mediastinum. The ultrasound image is dynamic rather than static and often presents anatomic relationships in planes that are different from standard CT scanning. Because of operative experience, the thoracic surgeon may be in a favorable position to understand the anatomic relationships displayed during EBUS. Although major societies have recommended at least 40 to 50 supervised procedures to establish basic EBUS competency, this requirement may be less for surgeons who routinely perform bronchoscopy and mediastinal 
procedures. ${ }^{14,15}$ Emerging minimally invasive diagnostic modalities will continue to challenge established approaches. To remain at the forefront of the diagnosis, staging, and management of lung cancer, surgeons should invest the time and effort necessary to evaluate new technology firsthand.

\section{References}

1. Herth FJ, Krasnik M, Yasufuku K, Rintoul R, Ernst A. Endobronchial ultrasoundguided transbronchial needle aspiration. J Bronchol. 2006;13:84-91.

2. Dooms C, Vansteenkiste J. Positron emission tomography in non-small cell lung cancer. Curr Opin Pulm Med. 2007;13:256-60.

3. Ung YC, Maziak DE, Vanderveen JA, Smith CA, Gulenchyn K, Lacchetti C, et al. Fluorodeoxyglucose positron emission tomography in the diagnosis and staging of lung cancer: a systematic review. J Natl Cancer Inst. 2007;99:1753-67.

4. Vilmann P, Puri R. The complete "medical" mediastinoscopy (EUS-FNA + EBUS-TBNA). Minerva Med. 2007;98:331-8.

5. Little AG, Rusch VW, Bonner JA, Gaspar LE, Green MR, Webb WR, et al. Patterns of surgical care of lung cancer patient. Ann Thorac Surg. 2005;80:2051-6.

6. Hammoud ZT, Anderson RC, Meyers BF, Guthrie TJ, Roper CL, Cooper JD, et al. The current role of mediastinoscopy in the evaluation of thoracic disease. J Thorac Cardiovasc Surg. 1999;118:894-9.

7. Luke WP, Pearson FG, Todd TR, Patterson GA, Cooper JD. Prospective evaluation of mediastinoscopy for the assessment of carcinoma of the lung. $J$ Thorac Cardiovasc Surg. 1986;91:53-6.

8. Gephardt GN, Rice TW. Utility of frozen-section evaluation of lymph nodes in the staging of bronchogenic carcinoma at mediastinoscopy and thoracotomy. J Thorac Cardiovasc Surg. 1990;100:853-9.

9. Diacon AH, Schuurmans MM, Theron J, Louw M, Wright CA, Brundyn K, et al. Utility of rapid on-site evaluation of transbronchial needle aspirates. Respiration. 2005; 72:182-8.

10. Davenport RD. Rapid on-site evaluation of transbronchial aspirates. Chest. 1990; 98:59-61.

11. Yasufuku K, Nakajima T, Motoori K, Sekine Y, Shibuyan K, Hiroshima K, et al. Comparison of endobronchial ultrasound positron emission tomography, and CT for lymph node staging of lung cancer. Chest. 2006;130:710-8.

12. Yasufuku K, Nakajima T, Chiyo M, Sekine Y, Kiyoshi Shibuya K, Fujisawa T. Endobronchial ultrasonography: current status and future directions. J Thorac Oncol. 2007;2:970-9.

13. Herth FJF, Eberhardt R, Vilmann P, Krasnik M, Ernst A. Real-time endobronchial ultrasound guided transbronchial needle aspiration for sampling mediastinal lymph nodes. Thorax. 2006;61:795-8.

14. Ernst A, Silvestri GA, Johnstone D. Interventional pulmonary procedures: guidelines from the American College of Chest Physicians. Chest. 2003;123:1693-17.

15. Bollinger CT, Mathur PN. ERS/ATS statement on interventional pulmonology. Eur Respir J. 2002;19:356-73.

\section{Discussion}

Dr S. Swisher (Houston, Tex). Endobronchial ultrasonography is a novel minimally invasive technology that allows diagnosis and staging of mediastinal nodes through ultrasound-guided fine-needle aspiration. Dr Gilbert and colleagues are to be commended for rapidly incorporating this new technology into the thoracic surgeon's armamentarium and reviewing their preliminary results. It is critical to our profession that thoracic surgeons remain at the vanguard in learning this technique and assessing its strengths and weaknesses compared with other standard techniques, such as mediastinoscopy, so that as a profession, we remain included in the process of evaluating and staging lung cancer in patients.

My first questions revolve around diagnostic accuracy. Seventyfive patients were reviewed in this series for a variety of reasons, including diagnosis of malignancy and staging of lung cancer. The authors found that rapid on-site assessment was diagnostic only $69 \%$ of the time, whereas final cytologic evaluation was diagnostic $74 \%$ of the time. The authors could not identify any factors that were associated with improved diagnostic accuracy, including operator experience or number of lymph node stations sampled. In most series the diagnostic yield is higher than what the authors report here. How do the authors explain their diagnostic yield of $74 \%$ ?

Most studies have also demonstrated that the number of aspirations of each nodal station correlates with accuracy. How many passes were performed in this study at each nodal station, and could this have influenced diagnostic yield?

Lymph node size has also been found to correlate with diagnostic yield in several studies. Did the authors evaluate lymph node size with respect to the sampling accuracy, and did this correlate with diagnostic yield?

Finally, of note, $56 \%$ of the patients in this study had only 1 nodal station sampled. Why did the authors not sample more lymph nodes stations? Was it not possible in some of these patients?

Dr Gilbert. I will attempt to answer all of your pertinent questions in the sequence they were posed. The diagnostic yield of EBUS can be influenced significantly by the definition of a "diagnostic sample"' and the prevalence of cancer in the population studied. We have used stringent definitions of what is considered "diagnostic", when compared with other published series of EBUS. For instance, specimens interpreted as "suspicious for malignancy" or " atypical cells present" were not considered diagnostic because, in our opinion, clinical decisions cannot be safely based on such results.

The setting of your EBUS review can be used as an example to illustrate my second point regarding diagnostic yield. If a study of EBUS is carried out in a large cancer center, it is possible that a significant proportion of the patients will have mediastinal adenopathy of malignant cause. The nature of the cancer center's referral pattern would likely bias the study population in that manner. When using a highly specific diagnostic test, such as EBUS-TBNA, the diagnostic yield will automatically appear better in study populations with higher cancer prevalence. This epidemiologic phenomenon alone will result in improved diagnostic yield regardless of other factors (eg, lymph node size, number of needle passes, operator experience, and cytology expertise). The prevalence of cancer in our study was relatively low compared with other series, and this probably had an impact on our diagnostic yield. The diagnostic performance of EBUS at our institution is actually comparable to results previously published in the Journal of the American Medical Association by Dr Wallace et al from the Mayo Clinic. In the hands of these investigators, EBUS-TBNA alone had a lower sensitivity, specificity, and positive predictive value and a higher false-negative rate than what we have obtained in our initial experience. Because the prevalence of cancer in their study group and ours was similar, the diagnostic performance parameters should be comparable.

With regard to the number of needle passes per lymph nodes, we did not look at this data specifically. We routinely use 3 passes per lymph node unless a positive cancer diagnosis is obtained with a lesser number of passes. In general, if the rapid on-site cytologic evaluation remains nondiagnostic after 3 to 4 needle passes, we move on to the next lymph node station to be sampled or perform a mediastinoscopy when appropriate. 
With regards to lymph node size, patients were stratified as having lymph nodes smaller or equal to $1 \mathrm{~cm}$ in their shortest axis or larger than $1 \mathrm{~cm}$. There was no difference in diagnostic yield between those 2 subgroups.

You also asked why $56 \%$ of the patients did not have multistation sampling as is routinely done during mediastinoscopy. Our study sample included a heterogenous group of patients thought to be reflective of general thoracic surgery practice. For instance, we evaluated patients with a suspected recurrent cancer in a single abnormal mediastinal or hilar lymph node station on imaging, patients who were eventually diagnosed with small cell lung cancer, metastatic cancer, or sarcoidosis after the first station sampled, and patients with N3 disease. These clinical situations do not always require multiple stations to be sampled in order to obtain a diagnosis and stage, and to devise a treatment plan. In patients where the mediastinum is staged in preparation for lung resection, our approach is to evaluate bilateral paratracheal and subcarinal stations beginning with the N3 lymph node stations.

Dr Swisher. My second question revolves around the importance of using EBUS and pathologic staging to assess PET-positive mediastinal nodes. We are in an era similar to before when CT increase in size of greater than $1 \mathrm{~cm}$ was taken by the oncologist to mean positive nodes, often without pathology. Now we are doing the same thing with new technology, and it's critical that we can pathologically evaluate this. EBUS may be an avenue to allow pathologic confirmation. In the present series, diagnostic accuracy was only $67 \%$, with 29 of 43 PET-positive cases. In the 14 cases in which EBUS was nondiagnostic, was the node in question visualized and accessible by EBUS? Were these nodes in regions that would have been accessible by mediastinoscopy, or were they in the hilar or anteroposterior window regions?

Dr Gilbert. Four of these patients had a previous lung resection for lung cancer. Two underwent mediastinoscopy which was negative and 1 had a percutaneous fine-needle aspiration of a right paratracheal node which was positive for recurrent cancer. The fourth patient's follow-up PET scan showed resolution of the mediastinal FDG uptake and he was obsereved. Four additional patients had abnormal lymph node stations not accessible by mediastinoscopy. The remaining 6 patients had PET-positive mediastinal lymph nodes. Three underwent mediastinoscopy and it was positive for cancer in 2 and negative in 1 . The latter patient had an EBUSTBNA which was suspicious for lymphoma. The fourth patient had transbronchial biopsy of his lung mass which showed necrotizing granulomata. This type of biopsy was not considered an acceptable reference standard for EBUS as stated in our methods. The fifth patient had lung cancer and had previously received thoracic radiotheraphy. He was deemed a non-surgical candidate and declined to have more invasive diagnostic procedures performed. The last patient had a MRI scan for shoulder symptoms which revealed a scapular metastasis. He did not require any further mediastinal workup.

Dr Patterson. Let me just interrupt. In the interest of time, can we have minimal commentary and maximal short questions because we have a couple of other discussants who want to ask some questions.

Dr Swisher. Thank you for bringing these preliminary results to the forefront of our group.

Dr Gilbert. Thank you, Sir.

Dr R. Cerfolio (Birmingham, Ala). What was considered a positive PET? Did you use an absolute value of the node, ratio of the node to the primary tumor?

Dr Gilbert. Given the retrospective study design, the radiology reports were reviewed to determine whether or not a given site was positive or negative on PET. Standard uptake values are not always reported at our institution. From my discussions with nuclear medicine specialists and radiologists, a baseline level of FDG uptake is usually established, using the liver parenchyma for instance, and abnormal levels of FDG avidity are established in relationship to that baseline in each patient.

Dr Cerfolio. Okay - not ideal, was it. My second question is about the logistics and the politics. To keep everybody happy, the surgeons, pulmonologists, and pulmonary fellows, how are you doing it so the pulmonary fellows can come through to keep the pulmonologists happy, because they also control our volume, and the cardiothoracic fellows; how are you doing it at your institution to keep everybody happy?

Dr Gilbert. For those who wish to use the EBUS scopes acquired by the operating room, we have established basic credentialing requirements, which include completion of an off-site EBUS course and at least 5 on-site proctored cases. In our group, there is a pulmonologist who performs and teaches the procedure routinely. I believe that people who share a common interest in the diagnosis and treatment of lung cancer and mediastinal disease should consider learning the technique, regardless of their specialty.

Dr A. Vaporciyan (Houston, Tex). We have struggled with how many proctored EBUSs are required before one is considered trained in this. Obviously everybody has different definitions. Where did you come up with 5 proctored EBUSs to consider yourself trained in it? Was it from literature or your experience?

Dr Gilbert. It is an institution-specific policy which was established after discussion with the departmental chair. The literature suggest that 30-50 supervised procedures may be needed for pulmonologists to establish proficiency. This number may be lower for thoracic surgeons given their clinical training and operative experience with lung cancer and mediastinal diseases. 